\title{
Predictors of future microalbuminuria in children and adolescents with type 1 diabetes mellitus in Egypt
}

\author{
Safinaz El Habashy ${ }^{1}$, Amira Abd El Monem Adly ${ }^{1}$, Mohamed Salah Eldin Mohamed Abdel Kader ${ }^{2}$, \\ Sherine El-Tokhy Ali²
}

\begin{abstract}
${ }^{1}$ Department of Paediatrics, Faculty of Medicine, Ain Shams University, Cair, Egypt ${ }^{2}$ Department of Paediatrics, Faculty of Medicine, Misr University of Science and Technology (MUST), Giza, Egypt
\end{abstract}

Submitted: 22 July 2019

Accepted: 19 November 2019

Arch Med Sci Atheroscler Dis 2019; 4: e286-e297

DOI: https://doi.org/10.5114/amsad.2019.91433

Copyright (c) 2019 Termedia \& Banach

\author{
Corresponding author: \\ Mohamed Salah Eldin \\ Mohamed Abdel Kader \\ Department of Paediatrics \\ Faculty of Medicine \\ Misr University of \\ Science and Technology \\ (MUST) \\ Giza, Egypt \\ E-mail: kader48@hotmail.com
}

\begin{abstract}
Introduction: The present study was designed to assess the validity and efficacy of urinary markers (NAG, RBP, transferrin, $\alpha 1$-microglobulin, and plasma homocysteine) as early predictors of microalbuminuria in diabetic nephropathy in children and adolescents with type-1 diabetes, and its relation with haemoglobin glycated $\left(\mathrm{HbA}_{1 \mathrm{c}}\right)$, serum lipid profile, and blood pressure.

Material and methods: This study is a follow-up study to the 2002 study by Salem et al. The present study included 35 type 1 diabetes mellitus (T1DM) children and adolescents recruited from regular attendees of the specialised Diabetology Clinic, Children's hospital, Ain Shams University, with previously measured urinary $N$-acetyl- $\beta$-glucosaminidase (13) or homocysteine (11) or transferrin (28) or $\alpha 1$-microglobulin (27) or retinol binding protein (13) as an early predictor of diabetic nephropathy in T1DM. Thirty-five patients with type 1 diabetes mellitus were enrolled, and 24 patients were normoalbuminuric at baseline. The patients were tested for markers other than urinary microalbumin, to predict diabetic nephropathy and early renal impairment in children and adolescents with type 1 diabetes mellitus.

Results: Regarding the metabolic control between the studied groups, we found that there is significant difference in $\mathrm{HbA}_{1 c}$ between the microalbuminuric patients and the normoalbuminuric patients. According to the number of positive markers of diabetic nephropathy, the only parameter that was higher in patients with more than one elevated marker was mean systolic blood pressure. Although mean diabetic blood pressure was higher, it was not statistically significant. Regarding to the predictability of urinary markers, urinary $N$-acetyl- $\beta$-glucosaminidase is the most predictable marker with high sensitivity and specificity. The least sensitivity noticed was urinary RBP and the least specificity noticed was urinary $\alpha 1$-microglobulin.

Conclusions: Regarding the predictability of urinary markers, urinary NAG is the most predictable marker with both high sensitivity and specificity, with a sensitivity of $60 \%$, specificity $75 \%$, positive predictive value $60 \%$, negative predictive value $75 \%$, and a diagnostic accuracy of $0.58 \%$. Urinary RBP is another marker with low sensitivity but high specificity. Urinary $\alpha 1$-microglobulin is a valid marker with high sensitivity but low specificity. Contrary to previous markers, plasma homocysteine has high specificity but low sensitivity.
\end{abstract}

Key words: plasma homocysteine, urinary $N$-acetyl- $\beta$-glucosaminidase, transferrin, $\alpha 1$-microglobulin, retinol binding protein, micro albuminuria, diabetes mellitus, diabetic nephropathy. 


\section{Introduction}

Type 1 diabetes mellitus (T1DM) is a multisystem disease with both biochemical and anatomical/structural consequences. It is a chronic disease of carbohydrate, fat, and protein metabolism caused by the lack of insulin, which results from the marked and progressive inability of the pancreas to secrete insulin because of autoimmune destruction of the $\beta$ cells [1].

T1DM can occur at any age. It occurs most commonly in juveniles but can also occur in adults, especially in those in their late 30s and early 40s [2].

Diabetic complications can be classified broadly as microvascular or macrovascular disease. Microvascular complications include neuropathy (nerve damage), nephropathy (kidney disease), and vision disorders (e.g. retinopathy, glaucoma, cataract, and corneal disease). Macrovascular complications include heart disease, stroke, and peripheral vascular disease (which can lead to ulcers, gangrene, and amputation). Other complications of diabetes include infections, metabolic difficulties, impotence, autonomic neuropathy, and pregnancy problems [1].

Diabetic nephropathy is one of the most common microvascular complications of diabetes mellitus, greatly affecting the life quality and survival of the patients. Regarding global prevalence, the number of patients with diabetic nephropathy is increasing day by day [3]. The natural history of diabetic nephropathy is one of clinical silence for years to decades, during which time serious underlying renal lesions may be developing. Once the clinical manifestations, including the development of persistent microalbuminuria, are present the structural injury is often far advanced. Because interventions at these late stages of disease may only slow but not completely arrest the inexorable progression towards renal failure, understanding early natural history becomes important [4].

Diabetic nephropathy, one of the leading causes of end-stage renal disease, affects $20 \%$ to $30 \%$ patients with T1DM. The course of diabetic nephropathy is slow. An increased urinary albumin excretion rate of 30 to $300 \mathrm{mg} /$ day (microalbuminuria) constitutes an early stage of nephropathy, especially when it becomes persistent (at least two of three consecutive urine samples. Annual screening for microalbuminuria should be initiated once the child is 10 years of age and has had diabetes for 5 years; more frequent testing is indicated if values are increasing [5].

Screening for subclinical retinopathy, neuropathy, and nephropathy should be started at puberty and at least three years after the diabetes diagnosis with the goal of detecting early abnormalities responsible for subclinical disorders that can be reversed by improved metabolic control, thus pre- venting the occurrence of irreversible, potentially incapacitating lesions [6].

The association of well-established risk markers and promoters of renal injury, including the degree and tracking of albuminuria, glycaemic control, blood pressure changes, incipient retinopathy and genetic nephropathy, and the decision to start pharmacological intervention [7].

Increased urinary protein excretion in patients with diabetes has long been known to predict increased mortality, and its absence is associated with near-normal life expectancy. Recent studies confirmed and extended these findings by illustrating the progressive increase in mortality by degree of albuminuria. The excess mortality is due primarily to end-stage renal disease and to cardiovascular disease, which share many risk factor [8].

Urinary excretion of lower molecular weight proteins such as $N$-acetyl- $\beta$-glucosaminidase $(\beta-N A G)$ and retinol binding protein (RBP), $\alpha 1-m i-$ croglobulin, and transferrin (TRF) [9] indicate proximal tubular dysfunction and may identify diabetic patients at risk of developing diabetic nephropathy and may indicate the onset of microalbuminuria [10]. Also, plasma total homocysteine rises with increased urinary albumin excretion in diabetes [11].

The present study was planned to follow diabetic patients who were normoalbuminuric but had increased levels of other nephropathy markers, namely (plasma homocysteine, urinary $n$-acetyl- $\beta$-glucosaminidase, transferrin, $\alpha 1$-microglobulin, retinol binding protein) to ascertain the following: the lag period to develop microalbuminuria and the determinants of conversion to microalbuminuria.

\section{Material and methods}

\section{Subjects}

This study is a follow-up for el Salem et al., 2002. It included 35 type I diabetic children and adolescents recruited from regular attendees of the Diabetology Specialised Clinic, Children's Hospital, Ain Shams University, with previously measured urinary $N$-acetyl- $\beta$-glucosaminidase (13) or homocysteine (11) or transferrin (28) or $\alpha 1$-microglobulin (27) or retinol binding protein (13), as an early predictor of diabetic nephropathy in T1DM.

The study was a retrospective study including 35 patients with type I diabetes mellitus recruited from regular attendees of the Diabetes Clinic, Children's hospital, Ain Shams University during the period from October 1, 2011 to April 30, 2012.

Of the 35 diabetic patients who were enrolled, 24 patients were normoalbuminuric at baseline [9]. These patients were tested for markers other than urinary microalbumin, to predict diabetic 
nephropathy and early renal impairment in children and adolescents with T1DM by detection of the progression of microalbuminuria in children and adolescents without microalbuminuria. These markers are: $\alpha 1$-microglobulin, transferrin, RBP, NAG, and homocysteine.

They were 24 normoalbuminuric patients: 12 (50\%) males and 12 (50\%) females, their ages ranged from 12 to 33 years, with a mean age of $15.176 \pm 4.878$ years, and disease duration ranging from 3 to 17 years with a mean of $7.9 \pm 3.3$ years.

Five years or less from the onset of diabetes (end point), six patients turned microalbuminuric, whereas 18 developed excess microalbumin in urine after 5 years. Accordingly, sensitivity and specificity of urinary and plasma markers of diabetic nephropathy (DN) were calculated.

Arbitrarily, patients were divided into two groups according to level of urinary microalbumin: whether equal to or below $60 \mathrm{mg} / \mathrm{dl}$ (double the normal level of microalbuminuria (MA)) or above it. The two groups were compared with no significant difference in any parameter included.

They were divided according to positivity of markers of nephropathy:

- Urinary NAG: 10 patients T1DM without microalbuminuria with previously estimated NAG marker. Three of them had high level of NAG and seven had normal level of NAG.

- Urinary transferrin: 18 T1DM patients without microalbuminuria with previously estimated transferrin marker. All of them had high levels of transferrin.

- Urinary a1-microglobulin: 17 T1DM patients without microalbuminuria with previously estimated $\alpha 1$ microglobulin marker. Fourteen of them had high levels of $\alpha 1$-microglobulin and 3 patients had normal levels of $\alpha 1$-microglobulin.

- Urinary RBP: 10 T1DM patients without microalbuminuria with previously estimated RBP marker. Eight of them had high levels of RBP, and two patients had normal levels of RBP.

- Plasma homocysteine: 11 T1DM patients without microalbuminuria with previously estimated homocysteine marker. Three of them had high levels of homocysteine, and 8 patients had normal levels of homocysteine.

\section{Methods}

All the files of the patients were revised for the following:

1) Full medical history taking after informing them about the study and obtaining their consent to participate, laying stress on the following:

- Age of the patient at the time of the study.

- Age at the onset of diabetes.

- Duration of diabetes.
- Symptoms suggesting diabetic complications:

- Symptoms of hypertension as persistent headache.

- Swelling, tingling, and numbness of lower limbs.

- Urinary symptoms suggestive of urinary tract infection such as dysuria, frequency, and loin pain.

- Dose of insulin therapy calculated in (U/kg/day) during the year prior to the study.

2) Thorough clinical examination laying stress on: Blood pressure, measured by conventional mercurial sphygmomanometer, and hypertension defined as the median value more than the $95^{\text {th }}$ percentile of at least three independent measurements [12].

1) Anthropometry including:

- Height in meters by using a standard stadiometer.

- Weight in kg by using a mechanical column scale.

- Body mass index (BMI) calculated as percentage of: weight $(\mathrm{kg}) /$ height $\left(\mathrm{m}^{2}\right)$.

- Height and weight were expressed as percentile values.

2) Full neurological examination to detect peripheral neuropathy.

3) Fundus examination, to detect retinopathy by direct ophthalmoscopy. Laboratory investigations:

1) Glycosylated haemoglobin $\left(\mathrm{HbA}_{1 c}\right)$ by high-performance liquid chromatography using the BioRad haemoglobin testing system, D-10 Dual Program [13].

2) Microalbumin in urine: Urinary albumin excretion was measured using immune turbidimetric methods. It was used to assess the presence of nephropathy. Patients initially detected as having a urinary albumin excretion rate (AER) $>30 \mu \mathrm{g} / \mathrm{mg}$ creatinine were asked to perform three further urine collections at intervals of 3-6 months. Persistent microalbuminuria was defined when two of three samples showed an excretion rate of $30-300 \mu \mathrm{g} / \mathrm{mg}$ creatinine [14].

Serum cholesterol and serum triglycerides (TG):

1) Total cholesterol was assayed on a Synchron CX-9 system autoanalyser using the cholesterol esterase reaction applying a timed end-point method [15].

2) Triglycerides assay was done on a Synchron CX-9 autoanalyser using the lipase reaction applying a timed endpoint method [16].

Normal laboratory values of nephropathy markers: urinary NAG (5.95 U/gm), RBP (270 mg/ $\mathrm{dl})$, transferrin $(0.06 \mathrm{mg} / \mathrm{mmol}), \alpha 1$-microglobulin $(0.6 \mathrm{mg} / \mathrm{mmol})$, and plasma homocysteine (15 mmol/l), by ELISA technique [9]. 
Table I. Descriptive data of 24 diabetics with normoalbuminuria at baseline study

\begin{tabular}{|lc|}
\hline Parameter & Mean \pm SD (range) \\
\hline Age [years] & $12.7 \pm 4.4(4-19)$ \\
\hline Duration of DM [years] & $3.8 \pm 3.5(1-15)$ \\
\hline Height (\%) & $70 \pm 22.3(25-97)$ \\
\hline Weight (\%) & $71.5 \pm 20(25-97)$ \\
\hline SBP (\%c) & $61 \pm 13.6(50-90)$ \\
\hline DBP (\%) & $61 \pm 13.6(50-90)$ \\
\hline HbA $_{1 c}(\%)$ & $8.3 \pm 2.9(5-15)$ \\
\hline
\end{tabular}

\section{Statistical analysis}

Analysis of data was done by IBM computer using SPSS (statistical program for social science version 15.0) (SPSS Inc., Chicago, IL, USA) for Windows ${ }^{\circledR}$, as follows: description of quantitative variables as mean, SD, and range, description of qualitative variables as number and percentage and $\chi^{2}$ test was used to compare qualitative variables between groups. Fisher's exact test was used instead of $\chi^{2}$ when one expected cell or more was less than 5 . Unpaired $t$-test was used to compare two groups regarding quantitative variables. Paired $t$-test was used to compare quantitative variables in the same group.

One-way ANOVA test (analysis of variance) was used to compare more than two groups as regard quantitative variables. The Kruskal-Wallis test was used instead of one-way ANOVA for non-parametric data. Spearman's correlation test was used to rank different variables positively or inversely. Receiver operator characteristic curve (ROC) was used to find out the best cut of value and validity of certain variable. $P$-values $>0.05$ were considered insignificant, $p<0.05$ significant, and $p<0.01$ highly significant.

\section{Results}

Out of the 35 diabetics who were enrolled, 24 were normoalbuminuric. Table I describes clinical data of patients at baseline study (Figure 1). Patients at baseline were tested for markers other than microalbuminuria in urine to predict diabetic nephropathy, as shown in Table II. Patients were re-assessed at the time of study, and their clinical data are shown in Table III.

Table IV and Figure 2 show that with increasing duration, mean growth pattern percentiles declined.

At time of study, all patients turned to be microalbuminuric with variable onset and hence with duration as shown in Table V.

Arbitrarily, patients were divided into two groups according to the level of urinary microalbumin: equal to or below $60 \mathrm{mg} / \mathrm{dl}$ (double the normal level of MA) or above it. Patient groups were compared in Table VI, with no significant difference in any parameter included (Figures 3-5).

Patients were distributed according to onset of

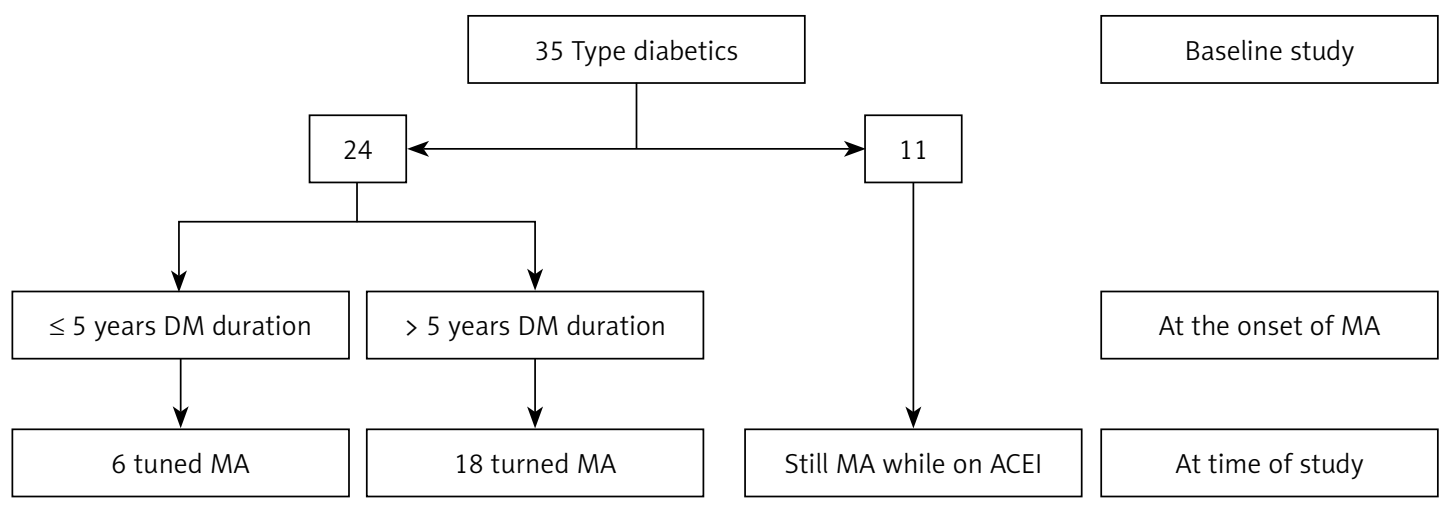

Figure 1. Outcomes of the 35 enrolled patients

Table II. Distribution of normoalbuminuric diabetics by markers at baseline

\begin{tabular}{|lccc|}
\hline Item & Normal, $n$ (\%) & High, $n$ (\%) & Total, $n$ (\%) \\
\hline Urinary NAG [U/gm] & $7(70)$ & $3(30)$ & $10(100)$ \\
\hline Urinary RBP [mg/dl] & $2(20)$ & $8(80)$ & $10(100)$ \\
\hline Urinary $\alpha$-microglobulin $[\mathrm{mg} / \mathrm{mmol}]$ & $3(30)$ & $14(82)$ & $17(100)$ \\
\hline Urinary transferrin $[\mathrm{mg} / \mathrm{mmol}]$ & $0(0)$ & $18(100)$ & $18(100)$ \\
\hline Plasma homocysteine $[\mathrm{mmol} / \mathrm{l}]$ & $8(80)$ & $3(27)$ & $11(100)$ \\
\hline
\end{tabular}


$M A$ and number of positive markers of MA into two groups as shown in Table VII (Figures 6-10).

\section{Discussion}

T1DM results from the autoimmune destruction of insulin-producing $\beta$-cells of the pancreas. Genetic and, as yet undefined, environmental factors act together to precipitate the disease. The excess mortality associated with the complications of T1DM and the increasing incidence of childhood T1DM emphasise the importance of therapeutic strategies to prevent this chronic disorder [17].

This study is a follow-up of 35 diabetic patients from the previous study of Salem et al. [9] as a baseline to our study. These patients were with previously measured five markers: $\alpha 1$-microglobulin, transferrin, retinol binding protein, homocysteine, $N$ acetyl glutaminase, and early renal impairment in children and adolescents with T1DM by detection of the progression of microalbuminuria in children and adolescents with microalbuminuria and those without.

It is a pioneer study in Egypt to measure these markers, and it was the baseline of our study to investigate the role of these marker in the prediction of microalbuminuria.

Diabetic nephropathy is a kidney disease that occurs as a result of diabetes. Cardiovascular and renal complications share common risk factors such as blood pressure, blood lipids, and glycaemic
Table III. Descriptive data of 24 diabetic patients at the time of study

\begin{tabular}{|lcc|}
\hline Parameter & Mean \pm SD & Range \\
\hline Age [years] & $25.2 \pm 4.9$ & $12-33$ \\
\hline Duration of DM [years] & $14.4 \pm 7.6$ & $1-25$ \\
\hline Weight (\%) & $81.9 \pm 14.7$ & $50-97$ \\
\hline Height (\%) & $83.7 \pm 14.6$ & $50-95$ \\
\hline BMl [kg/m²] & $31.6 \pm 6.1$ & $20-40$ \\
\hline SBP $(\%)$ & $87.9 \pm 10.8$ & $50-95$ \\
\hline DBP $(\%)$ & $72.8 \pm 18.1$ & $50-90$ \\
\hline HbA 1 (\%) & $12.6 \pm 3.3$ & $8-19$ \\
\hline Cholesterol $[\mathrm{mg} / \mathrm{dl}]$ & $267.2 \pm 72$ & $140-399$ \\
\hline Triglyceride $[\mathrm{mg} / \mathrm{dl}]$ & $144.4 \pm 50.6$ & $56-210$ \\
\hline
\end{tabular}

control. Thus, chronic kidney disease can predict cardiovascular disease in the general population. The impact of diabetes on renal impairment changes with increasing age. Serum markers of glomerular filtration rate and microalbuminuria identify renal impairment in different segments of the diabetic population, indicating that serum markers as well as microalbuminuria tests should be used in screening for nephropathy in diabetic people [18].

In our study there was correlation between microalbuminuria and the duration of diabetes. This

Table IV. Comparison between diabetics at the time of the study versus at baseline study

\begin{tabular}{|c|c|c|c|c|c|}
\hline \multirow[t]{2}{*}{ Parameter } & \multirow{2}{*}{$\begin{array}{c}\text { At study } \\
\text { Mean } \pm \text { SD }\end{array}$} & \multirow{2}{*}{$\begin{array}{c}\text { Baseline } \\
\text { Mean } \pm \text { SD }\end{array}$} & \multirow{2}{*}{$\begin{array}{l}\text { Difference } \\
\text { Mean } \pm \text { SD }\end{array}$} & \multicolumn{2}{|c|}{ Paired t-test } \\
\hline & & & & $T$ & $P$-value \\
\hline Age [years] & $25.2 \pm 4.9$ & $12.7 \pm 4.4$ & $12.5 \pm 3.5$ & 17.5 & $<0.001$ \\
\hline Duration [years] & $13.4 \pm 5.1$ & $3.8 \pm 3.5$ & $9.6 \pm 4.5$ & 10.4 & $<0.001$ \\
\hline Weight (\%) & $50.8 \pm 25.4$ & $71.5 \pm 19.9$ & $-20.7 \pm 23.1$ & -4.4 & $<0.001$ \\
\hline Height (\%) & $50.5 \pm 30.1$ & $69.9 \pm 22.3$ & $-19.4 \pm 31.4$ & -3.1 & 0.006 \\
\hline SBP (\%) & $82.4 \pm 11.4$ & $61.1 \pm 13.7$ & $21.4 \pm 17.1$ & 6.1 & $<0.001$ \\
\hline DBP (\%) & $78.5 \pm 12.2$ & $61.1 \pm 13.7$ & $17.5 \pm 19.1$ & 4.5 & $<0.001$ \\
\hline $\mathrm{HbA}_{1 \mathrm{c}}(\%)$ & $10.9 \pm 2.7$ & $8.3 \pm 2.9$ & $2.6 \pm 3.1$ & 3.7 & 0.001 \\
\hline
\end{tabular}

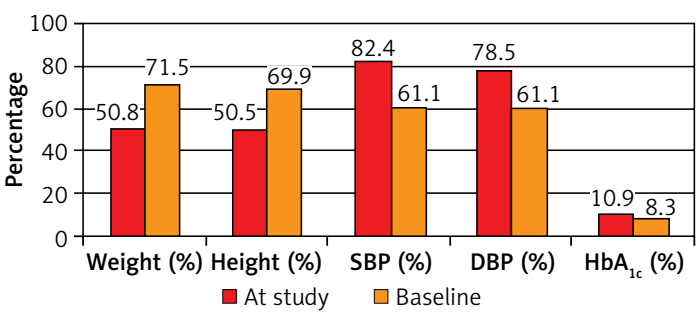

Figure 2. Clinical and laboratory parameters of diabetic patients at baseline study and at the time of the study
Table V. Onset and duration of MA in the studied diabetes

\begin{tabular}{|lcc|}
\hline Parameter & Mean \pm SD & Range \\
\hline Age at onset of MA [years] & $16.6 \pm 3.4$ & $8-22$ \\
\hline Duration of MA [years] & $7.9 \pm 3.3$ & $3-17$ \\
\hline
\end{tabular}


Table VI. Comparison between diabetics with high MA regarding their clinical and laboratory parameters

\begin{tabular}{|c|c|c|c|c|}
\hline \multirow[t]{2}{*}{ Parameter } & \multicolumn{2}{|c|}{ MA } & \multicolumn{2}{|c|}{$T$-test } \\
\hline & $\begin{array}{l}\leq 60 \mathrm{mg} / \mathrm{dl} \\
\text { Mean } \pm \text { SD }\end{array}$ & $\begin{array}{l}>60 \mathrm{mg} / \mathrm{dl} \\
\text { Mean } \pm \text { SD }\end{array}$ & $t$ & $P$-value \\
\hline Age of onset [years] & $12.9 \pm 4.3$ & $11.5 \pm 2.5$ & 0.7 & 0.39 \\
\hline Weight (\%) & $49.5 \pm 23.2$ & $53.0 \pm 30.2$ & -0.3 & 0.75 \\
\hline Height (\%) & $44.2 \pm 27.1$ & $61.1 \pm 33.5$ & -1.3 & 0.18 \\
\hline BMI $\left[\mathrm{kg} / \mathrm{m}^{2}\right]$ & $19.1 \pm 3.8$ & $19.1 \pm 2.5$ & -0.6 & 0.55 \\
\hline SBP (\%) & $79.9 \pm 13.1$ & $86.7 \pm 6.6$ & -1.4 & 0.16 \\
\hline DBP (\%) & $76.0 \pm 13.6$ & $82.8 \pm 8.3$ & -1.3 & 0.19 \\
\hline $\mathrm{HbA}_{1 \mathrm{c}}(\%)$ & $10.8 \pm 2.4$ & $11.1 \pm 3.2$ & -0.2 & 0.79 \\
\hline Cholesterol [mg/dl] & $178.4 \pm 34.6$ & $185.5 \pm 44.4$ & -0.4 & 0.66 \\
\hline Triglyceride [mg/dl] & $80.9 \pm 30.4$ & $99.8 \pm 38.6$ & -1.3 & 0.19 \\
\hline
\end{tabular}

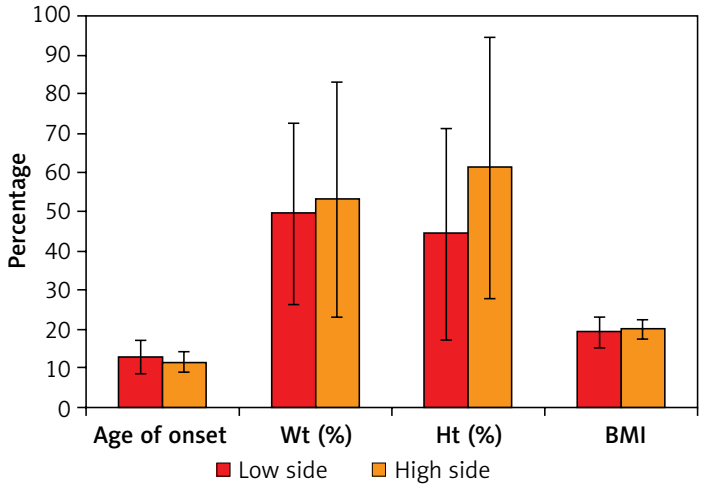

Figure 3. Clinical parameter of microalbuminurics with respect to MA level

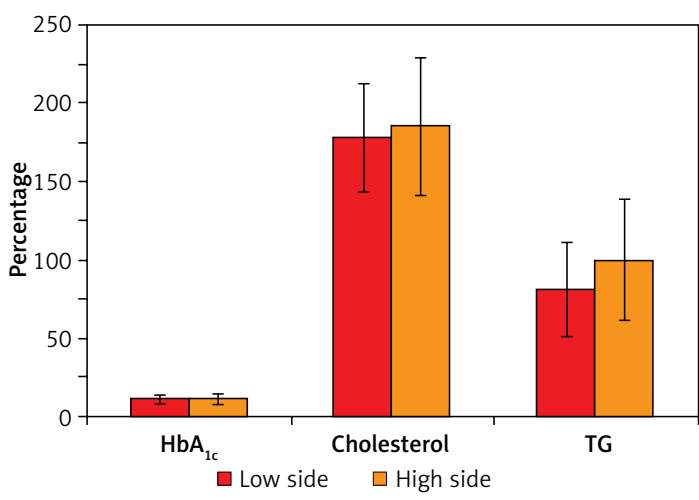

Figure 5. Laboratory parameter of microalbuminurics with respect to MA level

is in accord with Chowta et al. [19], who showed that the Incidence of microalbuminuria increases with age as well as with increased duration of diabetes mellitus. Earlier studies have shown positive correlation of microalbuminuria with the age of patients [20]. In our study there was no statistically significant age difference in the diabetic patients with and without microalbuminuria.

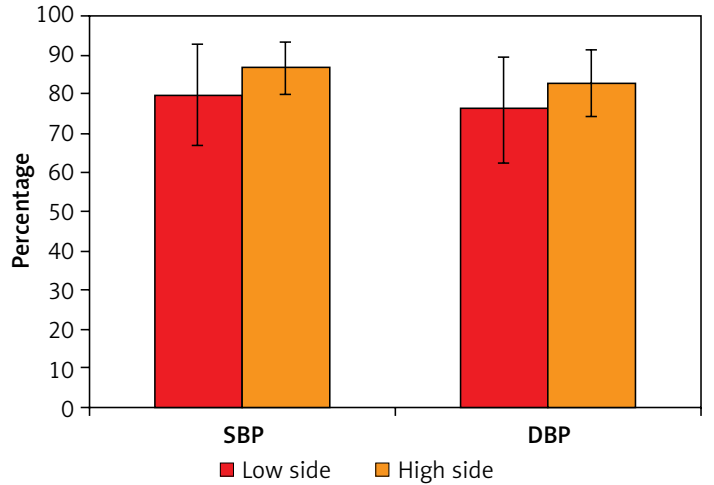

Figure 4. Clinical parameter of microalbuminurics with respect to MA level

Our study showed insignificant sex difference, and there was no significant difference in BMI between diabetic patients with or without microalbuminuria. This is in accord with Chowta et al. [19], who found that there was no effect of $\mathrm{BMI}$ and sex on the prevalence of microalbuminuria .

Our study included diabetic patients with positive microalbuminuria and systolic and diastolic blood pressure $>95^{\text {th }}$ percentile, but 11 of them had blood pressure $<95^{\text {th }}$ percentile because they were controlled by ACEI. This is in accordance with Gallego et al. [21] and Marcovecchio et al. [22] showing that that elevated blood pressure is a common finding in people with T1DM, and it has been associated with the risk of developing microvascular complications. In our study, patients with microalbuminuria had higher frequency of hypertension, with a statistically significant difference.

Among those with T1DM, the incidence of hypertension rises from $5 \%$ at 10 years, to $33 \%$ at 20 years, and $70 \%$ at 40 years. There is a close 
Table VII. Correlation between studied markers and lab values

\begin{tabular}{|lcccc|}
\hline Parameter & \multicolumn{2}{c|}{ Microalbuminuria at the baseline study } & \multicolumn{2}{c|}{ Microalbuminuria at follow-up } \\
\cline { 2 - 5 } & $r$ & $P$-value & $r$ & $P$-value \\
\hline NAG & 0.418 & 0.726 & 0.321 & 0.285 \\
\hline RBP & 0.839 & 0.367 & -0.378 & 0.203 \\
\hline$\alpha 1$-microglobulin & -0.234 & 0.514 & -0.078 & 0.699 \\
\hline Age & 0.544 & 0.084 & -0.027 & 0.881 \\
\hline Duration & 0.137 & 0.688 & 0.331 & $0.056^{*}$ \\
\hline BMI & -0.172 & 0.613 & 0.133 & 0.453 \\
\hline HBA ${ }_{1 c}$ & -0.021 & 0.951 & 0.318 & 0.067 \\
\hline Cholesterol & 0.222 & 0.512 & 0.272 & 0.119 \\
\hline Triglyceride & -0.027 & 0.938 & -0.066 & 0.709 \\
\hline Transferrin & -0.602 & $0.050^{*}$ & 0.159 & 0.368 \\
\hline
\end{tabular}

relationship between the prevalence of hypertension and increasing albuminuria. Blood pressure typically begins to rise within the normal range or within a few years after the onset of microalbuminuria and increases progressively as the renal disease progresses [23].

In our study, patients with microalbuminuria had higher frequency of complications, with a statistically significant difference in diabetics with positive microalbuminuria. It is either an acute complication (hypoglycaemia, diabetic ketoacidosis) or a chronic complication (microvascular com- plications such as retinopathy or neuropathy, and macrovascular complications such as coronary artery disease, cerebrovascular disease, peripheral vascular disease).

Girach and Vignati [24] showed that significant associations have been reported between the different microvascular complications of diabetes, so that patients with one complication often develop a second one, suggesting common risk factors and/or pathogenetic mechanisms.

We found that there was a significant correlation between serum cholesterol and serum

\begin{tabular}{|c|c|c|c|c|c|}
\hline \multicolumn{6}{|c|}{ ROC curve between negative and positive microalbuminuria } \\
\hline Cut-off & Sens. & Spec. & PPV & NPV & Accuracy \\
\hline$\leq 1.8$ & 50.0 & 82.4 & 62.5 & 73.7 & 0.682 \\
\hline
\end{tabular}
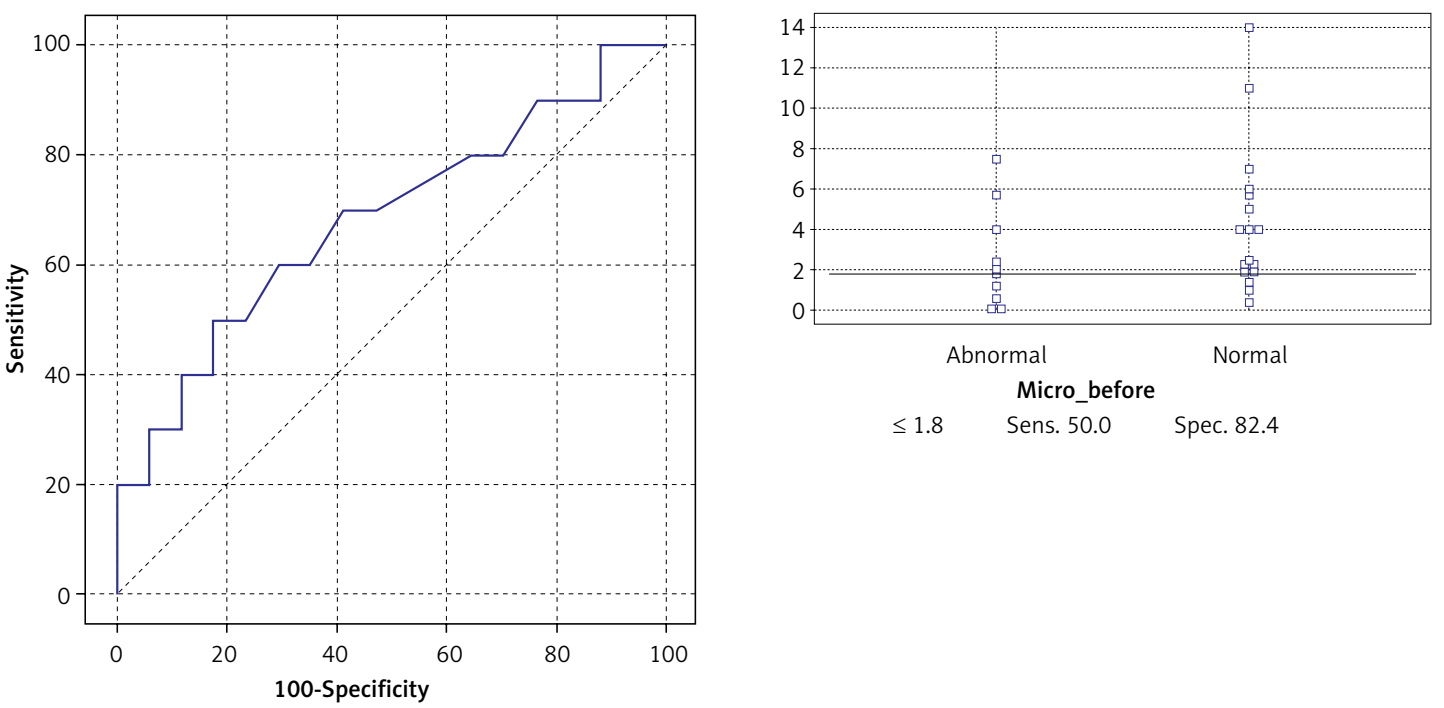

Figure 6. ROC curve to define the best cut-off $\alpha 1$-microglobulin $(\mathrm{mg} / \mathrm{l})$ to detect microalbuminuria. Urinary $\alpha 1$-microglobulin (mg/l) was a valid marker for microalbuminuria. The optimum cut-off value was $1.8 \mathrm{mg} / \mathrm{l}$, with a sensitivity of $50 \%$, specificity $82.4 \%$, positive predictive value (PPV) $55.6 \%$, and negative predictive value (NPV) $100 \%$ with a diagnostic accuracy of $0.63 \%$ 


\begin{tabular}{|c|c|c|c|c|c|}
\hline \multicolumn{6}{|c|}{ ROC curve between negative and positive microalbuminuria } \\
\hline Cut-off & Sens. & Spec. & PPV & NPV & Accuracy \\
\hline$\leq 400$ & 100.0 & 50.0 & 55.6 & 100.0 & 0.637 \\
\hline
\end{tabular}
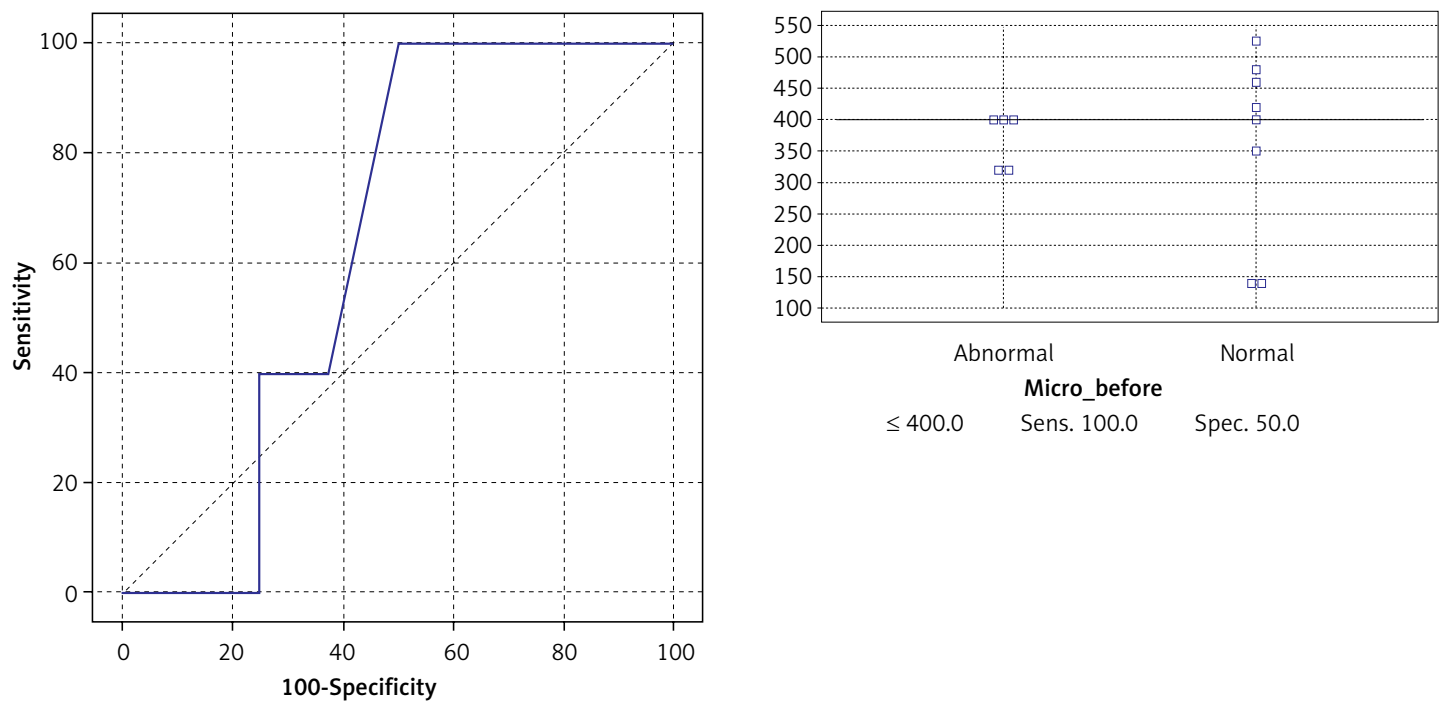

Figure 7. ROC curve to define the best cut-off. Retinol binding protein in $\mathrm{mg} / \mathrm{l}$ to detect microalbuminuria. Urinary retinol binding protein $(\mathrm{mg} / \mathrm{l})$ was a moderately valid marker for microalbuminuria. $\mathrm{P}<0.0001$ and area under the curve (AUC) was $99 \%$. The best cut-off value was $<400 \mathrm{mg} / \mathrm{l}$, with a sensitivity of $100 \%$, specificity $50 \%$, PPV $62 \%$, NPV $73 \%$, and diagnostic accuracy of $0.68 \%$

\begin{tabular}{|c|c|c|c|c|c|}
\hline \multicolumn{6}{|c|}{ ROC curve between negative and positive microalbuminuria } \\
\hline Cut-off & Sens. & Spec. & PPV & NPV & Accuracy \\
\hline$>10$ & 50.0 & 77.8 & 55.6 & 73.7 & 0.533 \\
\hline
\end{tabular}
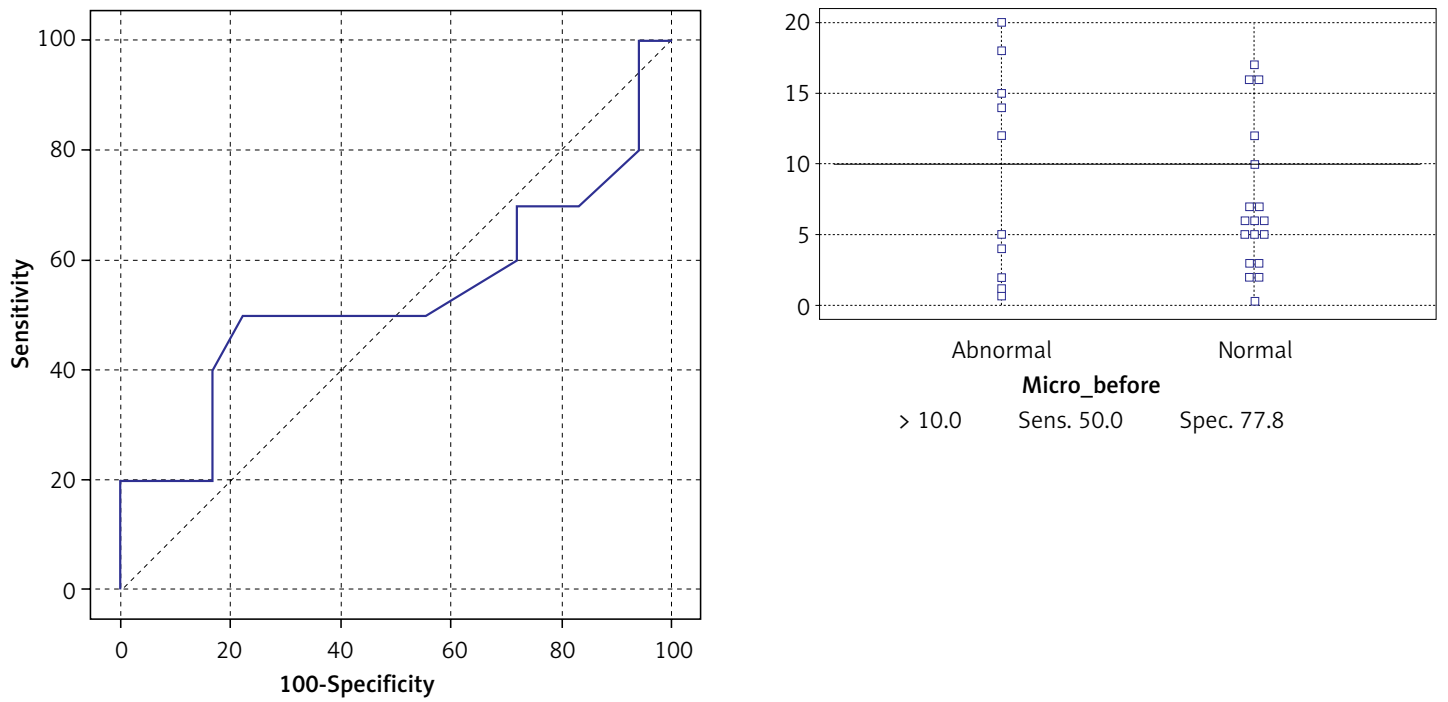

Figure 8. ROC curve to define the best cut-off transferrin $(\mathrm{mg} / \mathrm{l})$ to detect microalbuminuria. Urinary transferrin ( $\mathrm{mg} / \mathrm{l})$ was a moderately valid marker for microalbuminuria. The best cut-off value was $10 \mathrm{mg} / \mathrm{l}$, with a sensitivity of $50 \%$, specificity $77 \%$, PPV $55.6 \%$, NPV $73 \%$, and diagnostic accuracy of $0.533 \%$

triglyceride and microalbuminuria in diabetic patients. This is in accordance with other studies that show that chronic renal disease is accompanied by characteristic abnormalities of lipid metabolism are reflected in elevated plasma lipid levels [25].
In addition, Katakami et al. [26], in parallel to our study, showed that triglyceride levels were significantly higher in patients with T1DM compared to non-diabetic individuals $(p<0.05)$.

Our results disagree with those of Singh et al. [27] and $\mathrm{Oz}$ Gul et al. [28], who demonstrated that there 


\begin{tabular}{|c|c|c|c|c|c|}
\hline \multicolumn{6}{|c|}{ ROC curve between negative and positive microalbuminuria } \\
\hline Cut-off & Sens. & Spec. & PPV & NPV & Accuracy \\
\hline$\leq 4.3$ & 60.0 & 75.0 & 60.0 & 75.0 & 0.587 \\
\hline
\end{tabular}
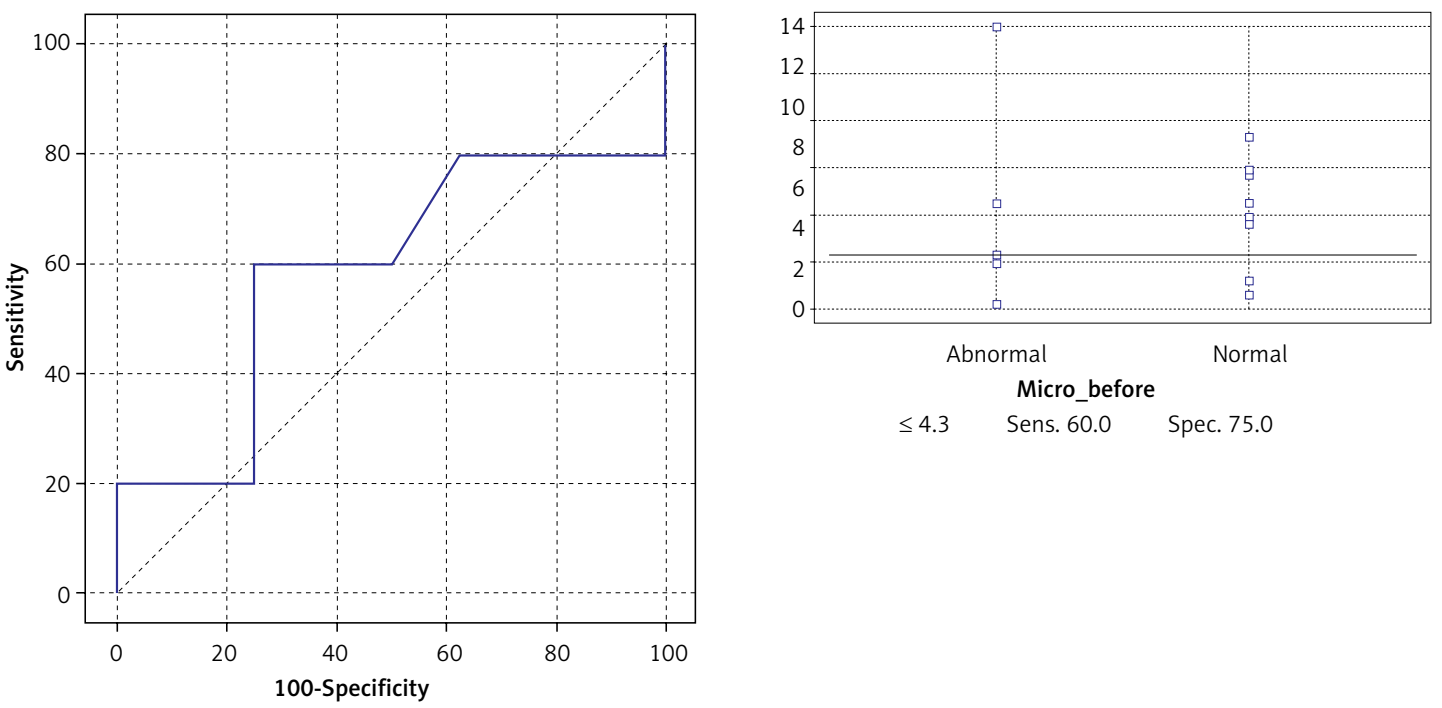

Figure 9. ROC curve to define the best cut-off urinary NAG $(\mathrm{mg} / \mathrm{l})$ to detect microalbuminuria. Urinary NAG (mg/l) is a valid marker for microalbuminuria $p<0.0001$ and AUC was $99 \%$. The best cut-off value was $<4.3 \mathrm{mg} / \mathrm{l}$, with a sensitivity of $60 \%$, specificity $75 \%$, PPV $60 \%$, NPV $75 \%$, and diagnostic accuracy of $0.58 \%$

\begin{tabular}{|c|c|c|c|c|c|}
\hline \multicolumn{6}{|c|}{ ROC curve between negative and positive microalbuminuria } \\
\hline Cut-off & Sens. & Spec. & PPV & NPV & Accuracy \\
\hline$>17.1$ & 18.2 & 100.0 & 100.0 & 72.7 & 0.551 \\
\hline
\end{tabular}
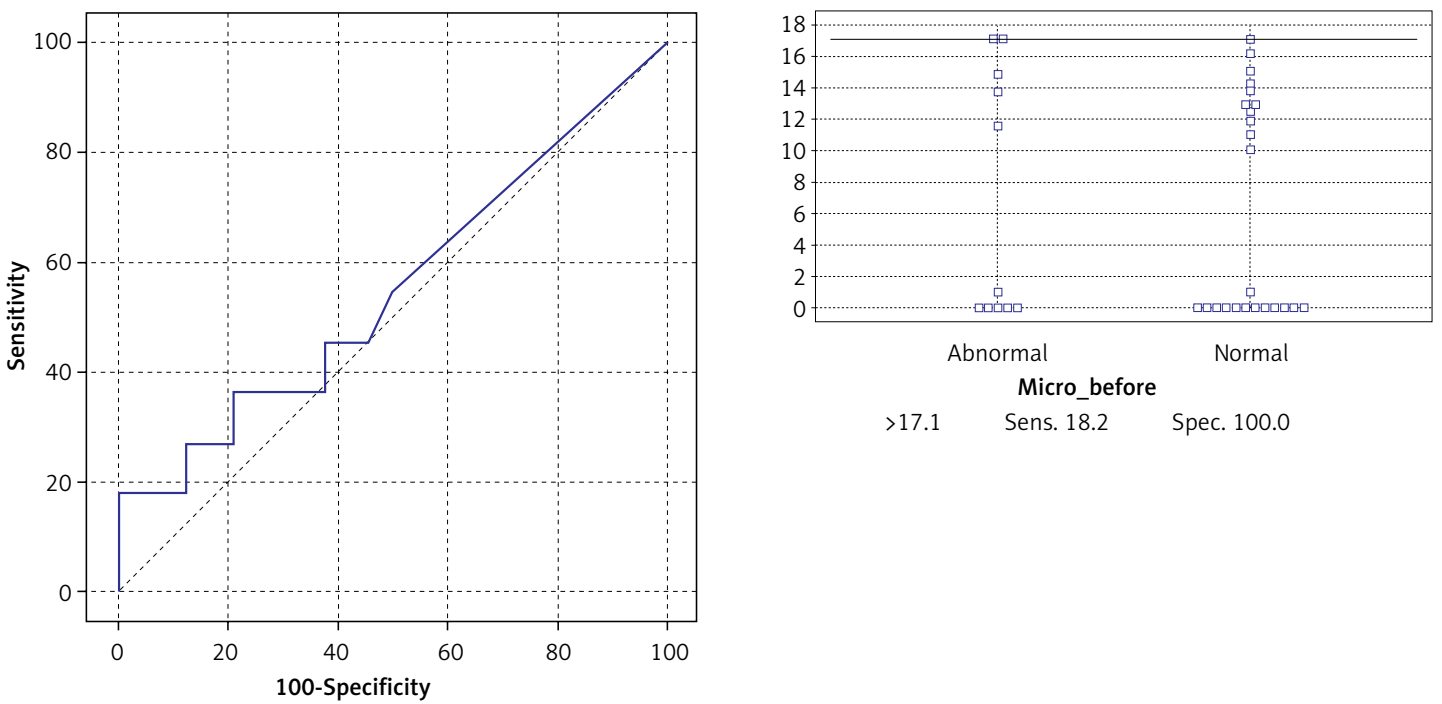

Figure 10. ROC curve to define the best cut-off homocysteine $(\mathrm{mg} / \mathrm{l})$ to detect microalbuminuria. Urinary homocysteine (in $\mathrm{mg} / \mathrm{L}$ ) was reliable in the prediction of microalbuminuria. The best cut-off value was $17.1 \mathrm{mg} / \mathrm{l}$, with a sensitivity of $18.2 \%$, specificity $100 \%$, PPV $100 \%$, NPV $72.7 \%$, and diagnostic accuracy of $0.55 \%$

was no difference in total cholesterol and triglycerides between diabetics with and without microalbuminuria.

There was no statistically significant difference between both groups regarding $\mathrm{HbA}_{1 c}(p=0.108)$, and this disagrees with the results of

Hovind et al. [1], who stated that increased $\mathrm{HbA}_{1 \mathrm{c}}$ as a marker of chronic hyperglycaemia is the most established and unquestioned risk factor for diabetic kidney disease in adult and paediatric T1DM, with $p<0.001$. This study included 286 participants, of whom 216 were adults.

Raile et al. [29] found that microalbuminuria was associated with this study included 27,805 children, adolescents, and adults with T1DM. 
One of limitation of our study was the small cohort.

This study evaluated the correlation between previously measured markers: $\alpha 1$-microglobulin, transferrin, retinol binding protein, homocysteine, $\mathrm{N}$ acetyl glutaminase, and early renal impairment in children and adolescents with T1DM, by detection of the progression of microalbuminuria in children and adolescents with microalbuminuria and those without, in the study done by Salem et al. [9]. We considered these markers as the baseline markers because this was a retrospective study of the same patients.

The ability to detect early and probably reversible renal injury using relatively inexpensive, non-invasive, and reliable biomarkers should lead to better care. Diabetic tubulointerstitial injury is a feature of early diabetic nephropathy and an important predictor of future renal dysfunction. Before the onset of gross structural changes in the renal tubules, lysosomal enzymes like NAG have been found to be markedly increased in urine. NAG marker is a significant marker (with mean \pm SD of $9.9933 \pm 3.7$ and a $p$-value of 0.011 ). It is a valid marker with $60 \%$ sensitivity and $75 \%$ specificity. Several studies elsewhere have shown increased urinary excretion of NAG in diabetics.

The results of our study confirm and extend the previous observations in small selected groups of patients with diabetes as in the baseline study of Salem et al. [9]. Our study included 13 patients with previously estimated NAG marker. Six of them had high levels of NAG and seven had normal levels of NAG. Three of the patients with high levels of NAG were positive for microalbuminuria and were still microalbuminuric, and the other three patients with high levels of NAG with negative microalbuminuria also developed microalbuminuria.

Salem et al. [9] showed that diabetic nephropathy (DN) is usually characterised by glomerular dysfunction, with microalbuminuria as an early indicator. Urinary excretion of lower molecular weight proteins such as $\beta$-NAG with mean \pm SD $6.88 \pm 3.76(p<0.001)$ and RBP with mean \pm SD $386.6 \pm 151.8(p<0.001)$ indicate proximal tubular dysfunction and may identify diabetic patients at risk of developing diabetic nephropathy.

This goes hand in hand with Vaidya et al. [30], who showed that low urinary levels of NAG are associated with regression of microalbuminuria in T1DM, suggesting that tubular dysfunction is a critical component of the early course of DN. One of the limitations of the study by Vaidya et al. [30] is the small number of patients who had microalbuminuria progression, thereby preventing adequate evaluation of biomarkers associated with microalbuminuria progression. Although I agree with Vaidya et al. [30], one of the advantages of our study is that we followed up the patients for 10 years whereas Vaidya et al. [30] followed up only for 2 years, which is too short to adequately determine the potential of urinary NAG to predict permanent regression or progression of microalbuminuria.

Another significant marker is transferrin (with mean \pm SD of $12.273 \pm 6.405$ ). It is a valid marker with moderate sensitivity of $50 \%$ and specificity of $77 \%$. Our study included 28 patients with previously estimated transferrin marker. All of them had high levels of transferrin; 10 patients with high levels of transferrin were positive for microalbuminuria and were still microalbuminuric, and the other 18 patients with high levels of transferrin and with negative microalbuminuria also developed microalbuminuria.

Transferrin is synthesised in liver. It carries less negative charge than microalbuminuria. When the glomerular charge barrier is impaired, transferrin will run into urine more easily than microalbuminuria. Transferrin is a sensitive indicator for glomerular charge barrier damage. When renal glomeruli are impaired, elevation of transferrin excretion occurs earlier than microalbuminuria.

The concentration of urine transferrin is lower than that of albumin. It may be degraded much more easily than albumin. Albuminuria and transferrin should be combined in early diagnosis of diabetic nephropathy [31].

Another significant marker is serum homocysteine level (with mean \pm SD of $1.33 \pm 3.95$ SD and $p$ $<0.001$ ). It is a highly valid marker with $18 \%$ sensitivity and 100\% specificity. Our study included 17 patients with previously estimated homocysteine marker. Seven of them had high levels of homocysteine, and 10 patients had normal levels of homocysteine. Three patients with high levels of homocysteine were positive for microalbuminuria and were still microalbuminuric, and the other four patients with high levels of homocysteine with negative microalbuminuria also developed microalbuminuria.

This agrees with the results of Stühlinger et al. [32], which showed that overt nephropathy is associated with elevations of plasma homocysteine in patients with diabetes, with a $p<0.05$.

Retinol binding protein (RBP) marker and $\alpha 1$-microglobulin marker are insignificant in the prediction of diabetic nephropathy.

RBP was with mean \pm SD of $44.2 \pm 72.7$ and $p=0.20$. RBP is a valid marker with $100 \%$ sensitivity and $50 \%$ specificity. Our study included 13 patients with previously estimated RBP marker. Eleven of them had high levels of RBP, and two patients had normal levels of RBP. Three patients with high levels of RBP were positive for microalbuminuria and still microalbuminuric, and the other 8 patients with high levels of RBP and with negative microalbuminuria also developed microalbuminuria. 
$\alpha 1$-microglobulin was with mean \pm SD of 4.86 \pm 3.7 and $p$ of 0.169 . $\alpha 1$-microglobulin is a valid marker with $50 \%$ sensitivity and $82 \%$ specificity. Our study included 27 patients with previously estimated $\alpha 1$-microglobulin. Twenty-two of them had high levels of $\alpha 1$-microglobulin, and five patients had normal levels of $\alpha 1$-microglobulin. Eight patients with high levels of $\alpha 1$-microglobulin were positive for microalbuminuria and were still microalbuminuric, and of the other 14 patients with high levels of $\alpha 1$-microglobulin who were negative for microalbuminuria, only 10 developed microalbuminuria.

This does not agree with Ching et al. [33], who showed that $\alpha 1$-microglobulin has been studied as a marker for renal tubular dysfunction before. Due to its stability at low $\mathrm{pH}$, its use has been suggested in screening for tubular abnormalities. Increased excretion of $\alpha 1$-microglobulin was found in the early course, while albumin excretion was still within normal range in the urine of type I patients. Non-correlation of albuminuria with a1-microglobulin excretion in T1DM may indicate whether the kidney impairment is severe or diffuse. Although urinary $\alpha 1$-microglobulin and albumin are related, in early nephropathy one may be present in the absence of the other. Hence, in addition to urinary albumin (which mainly measures glomerular function), urinary $\alpha 1$-microglobulin (which measures proximal tubular function) is useful for the early detection and monitoring of renal disease in diabetic subjects.

Gandhi et al. [34] also agreed with our study and showed that although microalbuminuria has been proposed as an early predictive biomarker of DN, it is clear that in the majority of patients it can regress to normoalbuminuria and in a minority it progresses to proteinuria.

This agrees with Matheson et al. [35], who provided a synopsis of urinary biomarkers that potentially provide a basis for the development of improved diagnostic tests. Three main pathways for the sourcing of potential makers have been identified: kidney damage, oxidative stress, and lowgrade inflammation including atherosclerosis and vascular damage. This review briefly presents some of the most relevant urinary biomarkers that may be used to monitor the development or progression of diabetes and its complications. In particular, biomarkers of renal dysfunction such as transferrin and $N$-acetyl- $\beta$-D-glucosaminidase might prove to be better than urinary albumin, the current gold standard, in the detection of incipient nephropathy and risk assessment of cardiovascular disease.

Regarding the predictability of urinary markers, urinary NAG is the most predictable marker with both high sensitivity and specificity, and with a sensitivity of $60 \%$, specificity $75 \%$, PPV $60 \%$,
NPV $75 \%$, and diagnostic accuracy of $0.58 \%$. Urinary RBP is another marker with low sensitivity but high specificity. Urinary $\alpha 1$-microglobulin is a valid marker with high sensitivity but low specificity. Contrary to previous markers, plasma homocysteine has a high specificity but low sensitivity.

It should also be noted that all of the previously mentioned markers are now easily available and cheap to perform in most laboratories and clinical pathology laboratories in Egypt and all around the world, hinting clearly at their cost effectiveness in early detection of diabetic micro albuminuria and diabetic nephropathies.

\section{Conflict of interest}

The authors declare no conflict of interest.

\section{References}

1. Hovind P, Joergensen L, Schmedes A, Parving HH. Vitamin D levels, microvascular complications, and mortality in type 1 diabetes. Diabetes Care 2011; 34: 1081-5.

2. Philippe MF, Benabadji S, Barbot-Trystram L. Pancreatic volume and endocrine and exocrine functions in patients with diabetes. Pancreas 2011; 40: 359-63.

3. Perrin N, Torbjörnsdotter T, Jaremko GA, Berg UB. Risk markers of future microalbuminuria and hypertension based on clinical and morphological parameters in young type 1 diabetes patients. Pediatr Diabetes 2010; 11: 305-1.

4. Steinke JM, Mauer M. Lessons learned from studies of the natural history of diabetic nephropathy in young type 1 diabetic patients. Pediatr Endocrinol Rev 2008; 5 Suppl 4: 958-63.

5. Samanta D, Mallika V, Yadav S. Early microalbuminuria in adolescent type 1 diabetic patients: experience from a pediatric endocrine clinic in a developing country. J Diabetes Endocrinol 2012; 3: 6-10.

6. Dorchy $H$. Screening for subclinical complications in children and adolescents with type 1 diabetes: experience acquired in Brussels. Rev Med Brux 2010; 31 (2 Suppl): S87-108.

7. Casani A, Bangstad HJ, Chiarelli F. Detection and management of diabetic glomerulopathy in children and adolescents with insulin-dependent diabetes mellitus: need for improved knowledge and better care. J Pediatr Endocrinol Metab 2000; 13: 467-74.

8. Orchard TJ, Secrest AM, Miller RG, Costacou T. In the absence of renal disease, 20 year mortality risk in type 1 diabetes is comparable to that of the general population: a report from the Pittsburgh Epidemiology of Diabetes Complications Study. Diabetologia 2010; 53: 2312-9.

9. Salem MA, El-Habashy SA, Saeid OM, el-Tawil MM, Tawfik PH. Urinary excretion of $n$-acetyl-beta-D-glucosaminidase and retinol binding protein as alternative indicators of nephropathy in patients with type 1 diabetes mellitus. Pediatr Diabetes 2002; 3: 520-3.

10. Myśliwiec $M$, Balcerska A, Zorena K, et al. Serum and urinary cytokine homeostasis and renal tubular function in children with type 1 diabetes mellitus. J Pediatr Endocrinol Metab 2006; 19: 1421-7.

11. Tarnow L, Grarup N, Hansen T, Parving HH, Pedersen O. Diabetic microvascular complications are not associated 
with two polymorphisms in the GLUT-1 and PC-1 genes regulating glucose metabolism in Caucasian type 1 diabetic patients. Nephrol Dial Transplant 2001; 16 1653-6.

12. Klemens R, Angela G, Sabine H, Antje H, Desiree D, Petra B. Diabetic nephropathy in children, adolescents and adults with type 1 diabetes. Diabetes Care 2007; 30: 2523-8.

13. Goldstein DE, Little RR, Wiedmeyer HM, England JD, McKenzie EM. Glycated hemoglobin: methodologies and clinical applications. Clin Chem 1986; 32 (10 Suppl): B64-70.

14. Viberti GC, Hill RD, Jarrett RJ, Argyropoulos A, Mahmud $\mathrm{U}$, Keen $\mathrm{H}$. Microalbuminuria as a predictor of clinical nephropathy in insulin-dependent diabetes mellitus. Lancet 1982; 1: 1430-2.

15. Dietschy JM, Weeks LE, Delente JJ. Enzymatic measurement of free and esterified cholesterol levels in plasma and other biological preparations using the oxygen electrode in a modified glucose analyzer. Clin Chim Acta 1976; 73: 407-14

16. McGowan JE Jr. Antimicrobial resistance in hospital organisms and its relation to antibiotic use. Rev Infect Dis 1983; 5: 1033-48.

17. Gillespie KM. Type 1 diabetes: pathogenesis and prevention. CMAJ 2006; 175: 165-170.

18. Bloomgarden ZT. American College of Endocrinology Pre-Diabetes Consensus Conference: part three. Diabetes Care 2008; 31: 2404-9.

19. Chowta NK, Pant P, Chowta MN. Microalbuminuria in diabetes mellitus: association with age, sex, weight, and creatinine clearance. Indian J Nephrol 2009; 19: 53-6.

20. Ruilope LM, Segura J. Predictors of the evolution of microalbuminuria. Hypertension 2006; 48: 832-3.

21. Gallego PH, Shephard N, Bulsara MK, et al. Angiotensinogen gene T235 variant: a marker for the development of persistent microalbuminuria in children and adolescents with type 1 diabetes mellitus. J Diabetes Complications 2008; 22: 191-8.

22. Marcovecchio ML, Giannini C, Widmer B, et al. C-reac tive protein in relation to the development of microalbuminuria in type 1 diabetes: the Oxford Regional Prospective Study. Diabetes Care 2008; 31: 974-6.

23. Weber KT. Integrative cardiorenal pathophysiology: a symposium presented at the Southern Society fo Clinical Investigation, February 25-27, 2010. Am J Med Sci 2010; 340: 23-4.

24. Girach A, Vignati L. Diabetic microvascular complications: can the presence of one predict the development of another? J Diabetes Complications 2006; 20: 228-37.

25. Svensson MK, Cederholm J, Eliasson B, Zethelius B, Gudbjörnsdottir S; Swedish National Diabetes Register. Clinical use and effectiveness of lipid lowering therapies in diabetes mellitus: an observational study from the Swedish National Diabetes Register. PLoS One 2011; 6: e18744.

26. Katakami N, Matsuhisa M, Kaneto $\mathrm{H}$, et al. Endogenous secretory RAGE but not soluble RAGE is associated with carotid atherosclerosis in type 1 diabetes patients. Diab Vasc Dis Res 2008; 5: 190-7.

27. Singh NP, Bansal R, Thakur A, Kohli R, Bansal RC, Agarwal SK. Effect of membrane composition on cytokine production and clinical symptoms during hemodialysis: a crossover study. Ren Fail 2003; 25: 419-30.

28. Oz Gul O, Tuncel E, Yilmaz Y, et al. Comparative effects of pioglitazone and rosiglitazone on plasma levels of soluble receptor for advanced glycation end products in type 2 diabetes mellitus patients. Metabolism 2010; 59: 64-9.

29. Raile K, Galler A, Hofer S, et al. Diabetic nephropathy in 27,805 children, adolescents, and adults with type $1 \mathrm{di}-$ abetes: effect of diabetes duration, $\mathrm{A} 1 \mathrm{C}$, hypertension, dyslipidemia, diabetes onset, and sex. Diabetes Care 2007; 30: 2523-8

30. Vaidya VS, Niewczas MA, Ficociello LH, et al. Regression of microalbuminuria in type 1 diabetes is associated with lower levels of urinary tubular injury biomarkers, kidney injury molecule-1, and N-acetyl-beta-D-glucosaminidase. Kidney Int 2011; 79: 464-70.

31. Perkovic V, de Zeeuw D, Mahaffey KW, et al. Canagliflozin and renal outcomes in type 2 diabetes: results from the CANVAS Program randomised clinical trials. Lancet Diabetes Endocrinol 2018; 6: 691-704.

32. Stühlinger MC, Tsao PS, Her JH, Kimoto M, Balint RF, Cooke JP. Homocysteine impairs the nitric oxide synthase pathway: role of asymmetric dimethylarginine. Circulation 2001; 104: 2569-75.

33. Ching YP, Leong VY, Wong CM, Kung HF. Identification of an autoinhibitory domain of p21-activated protein kinase 5. J Biol Chem 2003; 278: 33621-4.

34. Gandhi GR, Stalin A, Balakrishna K, Ignacimuthu S, Paulraj MG, Vishal R. Insulin sensitization via partial agonism of PPARgamma and glucose uptake through translocation and activation of GLUT4 in PI3K/p-Akt signaling pathway by embelin in type 2 diabetic rats. Biochim Biophys Acta 2013; 1830: 2243-55.

35. Matheson A, Willcox MD, Flanagan J, Walsh BJ. Urinary biomarkers involved in type 2 diabetes: a review. Diabetes Metab Res Rev 2010; 26: 150-71. 